\title{
Evaluation of low cost solutions for the transmission through optical fiber of low phase noise OCXO signals
}

\author{
S. Azaizia, K. Saleh*, O. Llopis \\ CNRS ; LAAS ; Université de Toulouse \\ 7 av. du Colonel Roche \\ 31077 Toulouse, France
}

*K. Saleh is also with CNES, Toulouse, France

\author{
A. Rissons \\ ISAE ; Université de Toulouse \\ 10 av. Edouard Belin, \\ BP 54032, 31055 Toulouse, France
}

\begin{abstract}
Various fiber optics links are evaluated for the transmission of OCXO signals on relatively short distances $(1 \mathrm{~m}$ to $1 \mathrm{~km}$ ). The case of VCSEL lasers is compared to the case of the more classical DFB laser. The DFB is still the best device, particularly in terms of signal to noise ratio, but single-mode VCSELs are becoming interesting as a low cost alternative to this device.
\end{abstract}

\section{INTRODUCTION}

Optical fiber is today an efficient technique for clock distribution, and numerous systems already use this technique for medium or short distances transmissions (ex : antennas networks), or even on long distance applications (ex : clock comparison). Most of these systems are based on costly laser modules, either amplitude modulated DFB lasers, which includes an optical isolator and temperature control, or high spectral purity lasers (coherent transmission). Vertical Cavity Surface Emitting Lasers (VCSELs) are low cost lasers, which are today available at long wavelengths, $1300 \mathrm{~nm}$ and $1500 \mathrm{~nm}$, and which may be used for communications. It is thus interesting to compare their performances for this application, and particularly for an operation on short or medium distances ( $1 \mathrm{~m}$ to $1 \mathrm{~km}$ ).

Another interest is for the power splitting required for a clock distribution on various receivers (ex : $10 \mathrm{MHz}$ reference signal distribution towards a set of 10 or more $\mathrm{HF}$ synthesizers). If this power split is realized in the optical domain, using a medium or high power laser and an optical power splitter, the noise floor in the RF domain increases rapidly because of the optical losses. Indeed, the quadratic detection at the photodiode level results in a $2 \mathrm{~dB}$ RF losses for each $1 \mathrm{~dB}$ optical losses (and, as an example, an optical splitting by 10 results in, at least, $20 \mathrm{~dB}$ RF losses). If the power splitting is performed in the RF domain, then the resulting signals can be used to modulate a set of VCSEL for the optical transmission. The relatively low power and low cost VCSEL laser is well fitted to this solution, in which a lower RF power is used to modulate the laser current.
Among these studies, the case of the transmission of a low phase noise $10 \mathrm{MHz}$ signal is particularly interesting. This signal is generally provided in a system by an oven controlled crystal oscillator (OCXO). The transmission of high spectral purity signals using fiber optics links has been described in many papers [1-5], and particularly the classical approach in which the reference signal directly modulates the current of a medium power DFB laser. The phase noise floor, for this type of link, is mainly related to the laser relative intensity noise (RIN) and the laser modulation index $m$, at least for a point to point link ( $m=\frac{\Delta P}{P}$, with $P$ being the average optical power and $\Delta P$ the maximum deviation from $P$ due to the modulation; $m \leq 1)$. In case of a division on a large number of receivers, the noise floor is increased due to the optical losses and it may degrade even further if the shot noise limit is reached. Moreover, on long fiber links (typically above $1 \mathrm{~km}$ ), some optical nonlinear effects may limit the phase noise performance [6] at high optical power. For these reasons, and because of the cost argument, the replacement of the DFB laser by a VCSEL device, which operates at lower power, must be investigated.

\section{DEVICES UNDER TEST AND MAIN EXPERIMENT}

We have studied four types of optical links at $10 \mathrm{MHz}$ using four different lasers :

1) A telecommunications grade laser module (Mitsubishi), based on a $20 \mathrm{~mW}$ DFB laser emitting at $1550 \mathrm{~nm}$, which includes an optical isolator, a temperature control and a photodiode.

2) A single mode VCSEL laser (Raycan) emitting about $0.5 \mathrm{~mW}$ at a wavelength of $1310 \mathrm{~nm}$.

3) A single mode VCSEL laser (Raycan) emitting about $0.5 \mathrm{~mW}$ at a wavelength of $1550 \mathrm{~nm}$.

4) A multimode VCSEL laser (Ulm Photonics) emitting about $10 \mathrm{~mW}$ at a wavelength of $850 \mathrm{~nm}$. 
The DFB laser module is ready to use : it includes an RF input which feeds an internal bias Tee. A dedicated test fixture has been designed for the three other devices, which includes a $10 \mathrm{MHz}$ bias Tee.

On the receiver side, an InGaAs photodiode (Thorlabs FGA04) is used to recover the RF signal. The photodiode delivers the RF current to a $50 \Omega$ load, through a bias Tee (Mini-Circuits). The same photodiode is used in the three experiments at large wavelength (1310 to $1550 \mathrm{~nm})$, and is replaced by a silicon photodiode for the $850 \mathrm{~nm}$ case (Thorlabs FDS02). The RF signal is measured at the photodiode output directly, or is amplified prior measurement if its level is close to (or below) $-20 \mathrm{dBm}$.

Figure 1 represents the schematics of the main experiment. A low phase noise $10 \mathrm{MHz}$ OCXO (Wenzel Premium SC50104609) is used to modulate the laser. The signal goes through a fiber spool and is detected by a photodiode and then, reaches the phase noise measurement system (Agilent E5052B cross-correlation phase noise analyser).

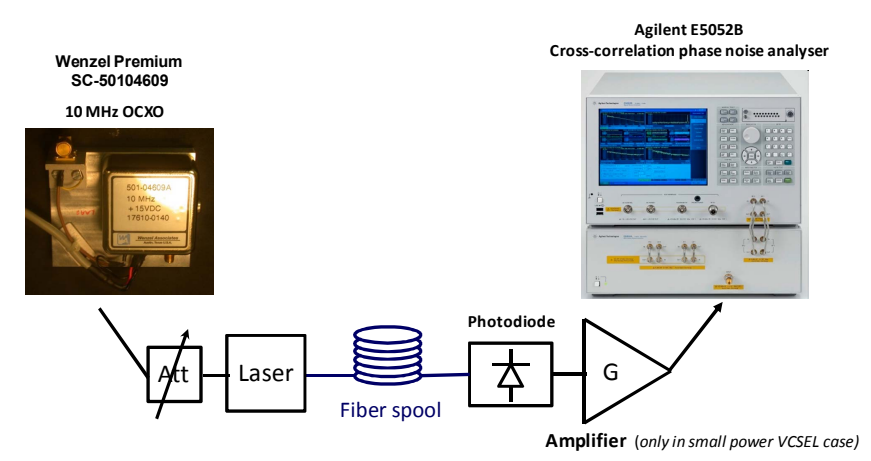

Figure 1. Schematics of the main experiment

\begin{tabular}{|l|l|l|}
\hline Frequency Offset & $\begin{array}{l}\text { Phase Noise } \\
\text { Datasheet }\end{array}$ & $\begin{array}{l}\text { Phase noise } \\
\text { Measured }\end{array}$ \\
\hline $10 \mathrm{~Hz}$ & $-130 \mathrm{dBc} / \mathrm{Hz}$ & $-132 \mathrm{dBc} / \mathrm{Hz}$ \\
\hline $100 \mathrm{~Hz}$ & $-155 \mathrm{dBc} / \mathrm{Hz}$ & $-155 \mathrm{dBc} / \mathrm{Hz}$ \\
\hline $1 \mathrm{kHz}$ & $-165 \mathrm{dBc} / \mathrm{Hz}$ & $-168 \mathrm{dBc} / \mathrm{Hz}$ \\
\hline $10 \mathrm{kHz}$ & $-165 \mathrm{dBc} / \mathrm{Hz}$ & $-170 \mathrm{dBc} / \mathrm{Hz}$ \\
\hline
\end{tabular}

Table 1: OCXO phase noise (measurement noise floor)

As it is not a residual phase noise measurement set-up, the phase noise floor is imposed by the OCXO phase noise (which is much lower than the optical links noise at carrier offsets above $100 \mathrm{~Hz}$ ). This noise floor is depicted in Table 1, with the typical values from Wenzel and the measured values on our oscillator.

For the single mode lasers (1310 nm and $1550 \mathrm{~nm})$, three different length of single mode fiber are used in this experiment : $1 \mathrm{~m}$ (no spool), $100 \mathrm{~m}, 1 \mathrm{~km}$. In the case of the multimode laser $(850 \mathrm{~nm})$, a $300 \mathrm{~m}$ multimode spool is used in place of the $100 \mathrm{~m}$ and $1 \mathrm{~km}$ spools.

\section{DFB LASER LINKS}

The DFB laser is biased at $80 \mathrm{~mA}$ and delivers an optical CW power of $17.8 \mathrm{~mW}$. The modulation index is set at 0.52 , in order to preserve the laser for long term operation. The RIN performance at $10 \mathrm{MHz}$ has been measured for this laser at $-162 \mathrm{~dB} / \mathrm{Hz}$. Under those conditions, the SSB phase noise floor is given by the noise to signal ratio divided two. The equation below [7] allows to compute this phase noise floor.

$$
L(f)=10 \log \left[\frac{10^{\frac{R I N}{10}}}{m^{2}}+\frac{2 q}{m^{2} I_{P D}}+\frac{4 k T}{R m^{2} I_{P D}^{2}}\right]
$$

with $L(f)$ being the $\mathrm{RF}$ phase noise in $\mathrm{dBc} / \mathrm{Hz}, R I N$ the laser relative intensity noise performance in $\mathrm{dB} / \mathrm{Hz}$ at the frequency of interest $(10 \mathrm{MHz}), m$ the RF modulation index, $I_{P D}$ the mean current at the photodiode output, $T$ the absolute temperature, $R$ the photodiode load, $q=1.610^{-19} \mathrm{C}$ and $k=1.3810^{-23} \mathrm{~m}^{2} \mathrm{~kg} \mathrm{~s}^{-1}$. In equation (1), the main contributor is the first term in case of a point to point link. The computed phase noise floor using (1) is very close to the one computed using only this term, and is about $-155 \mathrm{dBc} / \mathrm{Hz}$.

Concerning the $1 / \mathrm{f}$ phase noise, there is no simple analytical formula. This phase noise contribution comes from various nonlinear conversion phenomena, and particularly from the conversion of the laser $1 / \mathrm{f}$ RIN. The computation of the conversion coefficient would be beyond the scope of this paper, and this noise will be only qualitatively discussed.

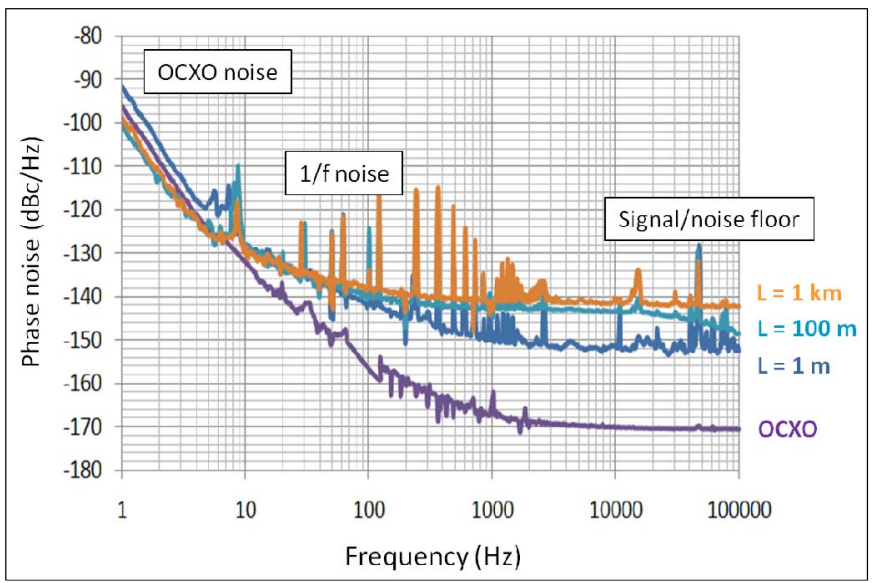

Figure 2. Phase noise at the output of the $10 \mathrm{MHz}$ optical links realized with the DFB laser module (and comparison to the OCXO phase noise)

Figure 2 depicts the measurements performed on the optical links realized with the DFB laser. The measurement noise floor (OCXO) is also depicted on the same graph. The measurement performed with $1 \mathrm{~m}$ fiber shows above $10 \mathrm{kHz}$ offset a white noise floor at about $-153 \mathrm{dBc} / \mathrm{Hz}$, which is close to the theoretical noise floor computed using equation (1). Between $10 \mathrm{~Hz}$ and a few hundred $\mathrm{Hz}$, a $1 / \mathrm{f}$ phase noise dominates this noise floor, and between $1 \mathrm{~Hz}$ and $10 \mathrm{~Hz}$, the measured phase noise at the output is the one of the OCXO. 
The case of the $100 \mathrm{~m}$ and $1 \mathrm{~km}$ links is a bit different. If the close to carrier phase noise has not changed compared to the $1 \mathrm{~m}$ case (OCXO noise below $10 \mathrm{~Hz}$ and $1 / \mathrm{f}$ noise between $10 \mathrm{~Hz}$ and $100 \mathrm{~Hz}$ ), the phase noise floor above $1 \mathrm{kHz}$ offset is much higher than the theoretically computed phase noise floor (about $10 \mathrm{~dB}$ higher). Actually, it is not exactly a noise floor, because in the $100 \mathrm{~m}$ case the phase noise drops again above $20 \mathrm{kHz}$ offset. This increase of the noise far from the carrier cannot be explained by a change in the signal to noise ratio. Indeed, the losses in $100 \mathrm{~m}$ or even $1 \mathrm{~km}$ of SMF28 fiber are almost negligible $(0.2 \mathrm{~dB} / \mathrm{km})$, even if the connector losses are included in the computation.

Therefore, a specific phenomenon occurs in long fibers. Two hypothesis may arise to explain this noise contribution. The first one is a conversion of the laser AM or, more probably, of its FM noise due to some specific phenomenon inside the long fiber (ex : frequency dispersion). The second one is the direct generation of a noise component inside the fiber.

The FM and AM noise of this laser have been measured, and a close to $1 / \mathrm{f}$ shape has been found on the $1 \mathrm{~Hz}$ to $100 \mathrm{kHz}$ frequency range (see Figure 5 for AM noise). Thus, such a noise contribution should be converted with a similar spectral shape, meanwhile a large flat is observed on the phase noise measurement. Therefore, the second hypothesis has been examined, and we have measured the noise due to the Rayleigh scattering in the fiber using a measurement approach described in details in [6]. The signal backscattered from the fiber is separated from the input signal using a circulator and is then mixed on a photodiode with the input signal. Figure 3 shows the result of this experiment performed with a $1 \mathrm{~km}$ fiber spool. It is clear that the same spectral shape is observed than in the phase noise measurement. Thus, this increased phase noise floor may be due to the Rayleigh scattering inside the fiber, and can be limited by reducing the optical power in the fiber (but in this case, a compromise has to be found with the signal to noise ratio).

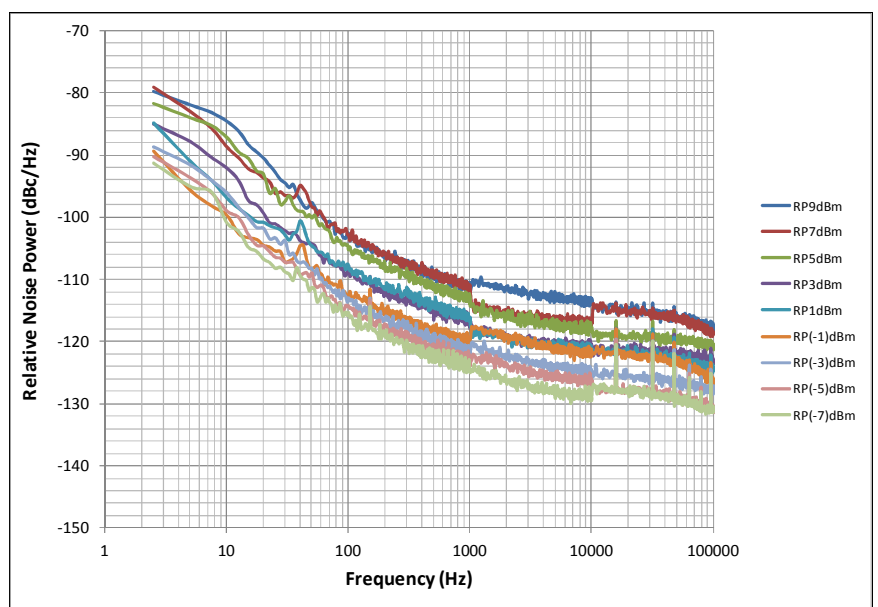

Figure 3. Measurement of the noise backscattered in the fiber (same method as in [7]) in the case of the $1 \mathrm{~km}$ spool and using the DFB laser. Each spectrum corresponds to a different input power inside the fiber.

\section{SINGLE MODE VCSELS LASER LINKS}

The link is then realized using a VCSEL (Raycan) in place of the DFB laser module. The laser is biased between $7 \mathrm{~mA}$ and $10 \mathrm{~mA}$, and delivers an optical power between $0.5 \mathrm{~mW}$ and $1 \mathrm{~mW}$ (maximum level, close to saturation). An RF attenuator is added between the OCXO and the laser, in order to adjust the laser modulation index, which is set between 0.55 and 0.7 (RF power in the range of $0 \mathrm{dBm}$ to $3 \mathrm{dBm}$ ). An optical isolator is also added at the laser output $(1550 \mathrm{~nm}$ laser). In the case of the $1310 \mathrm{~nm}$ laser, and because no isolator was available in the laboratory at this wavelength, the isolator has been replaced by a $3 \mathrm{~dB}$ optical attenuator.

The RF output power of such a link can be easily computed from the modulation index $m$, the received optical power $P_{o p t}$, the photodiode sensitivity $S_{P D}$ and the photodiode RF load $R$ using equation (2) :

$$
P_{R F o u t}=\frac{1}{2} R S_{P D}^{2} m^{2} P_{o p t}^{2}
$$

with $m=0.7, P_{\text {opt }}=1 \mathrm{~mW}, R=50 \Omega$ and $S_{P D}=0.8 \mathrm{~A} / \mathrm{W}$, the maximum RF power at the output of this optical link is found to be $-21 \mathrm{dBm}$. This level is of course much lower than the one obtained for the DFB link, in which the output RF power on the photodiode load is about $+2 \mathrm{dBm}$, under the conditions described in paragraph III. Because the phase noise measurement system requires a signal higher than $-20 \mathrm{dBm}$ at its input, an amplifier has been added between the photodiode and the analyser. The first measurements were performed with a commercial amplifier, and finally a specific amplifier has been designed for these links.

This amplifier uses a low $1 / \mathrm{f}$ noise silicon bipolar transistor BFR92, and has been optimized for $10 \mathrm{MHz}$ operation using Agilent ADS software. Particularly, an unbypassed emitter resistance $R_{e}$ of $8 \Omega$ has been used, both to stabilize the device and to reduce the amplifier phase noise. Finally, the amplifier has been realized and has demonstrated a gain of $19 \mathrm{~dB}$ at $10 \mathrm{MHz}$, together with a low residual phase noise, and particularly a noise floor of about $-165 \mathrm{dBc} / \mathrm{Hz}$.

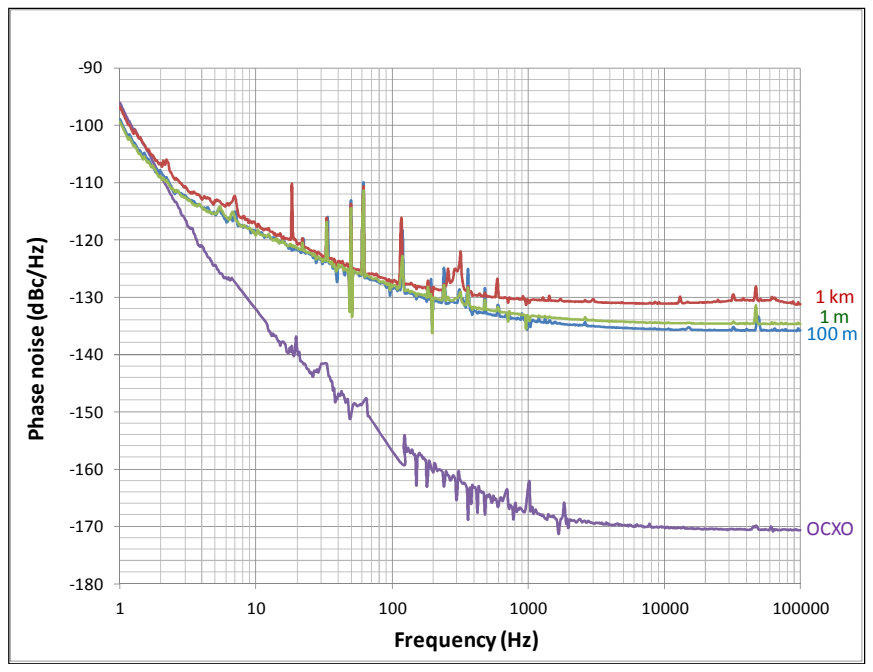

Figure 4. Phase noise at the output of the $10 \mathrm{MHz}$ optical links realized with the $1310 \mathrm{~nm}$ single mode VCSEL 
Figure 4 depicts the phase noise measured at the output of the optical link which uses the $1310 \mathrm{~nm}$ single mode VCSEL device. A noise floor in the range of $-135 \mathrm{dBc} / \mathrm{Hz}$ is observed. Between $3 \mathrm{~Hz}$ and $200 \mathrm{~Hz}$, the noise is clearly of 1/f type and, very close to the carrier $(1 \mathrm{~Hz})$, the OCXO phase noise is found again. The phase noise floor is close to what could be expected using equation (1) for this laser, although for the $1 \mathrm{~km}$ spool case, the noise is increased of about $4 \mathrm{~dB}$ compared to the two other cases. The high $1 / \mathrm{f}$ noise contribution is not too much surprising, because these lasers are small devices (with high current density) and thus feature a relatively high level of 1/f AM (and probably FM) noise. As an example, the VCSEL low frequency RIN is depicted in Figure 5, and compared to the same data measured on the DFB laser.

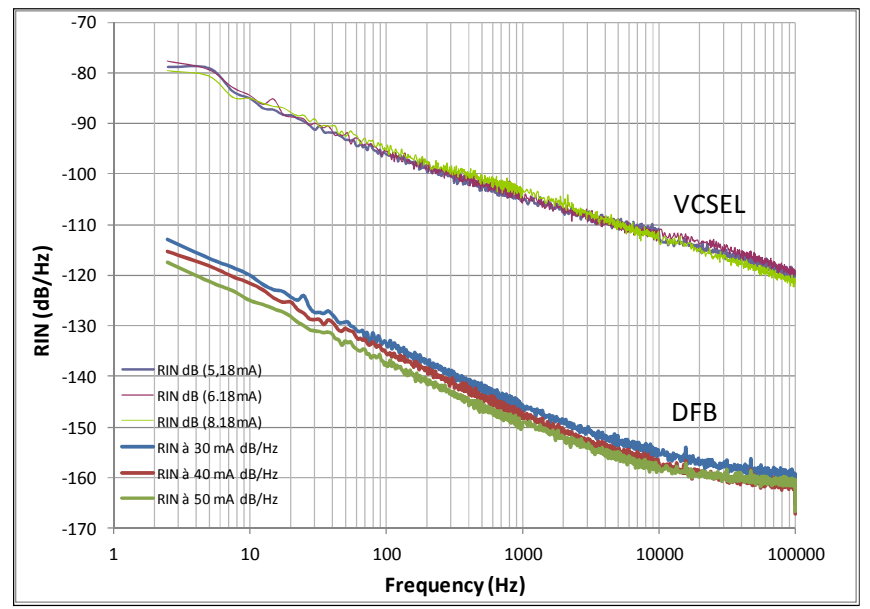

Figure 5. RIN performance measured at low frequency for the $1550 \mathrm{~nm}$ DFB laser and the $1310 \mathrm{~nm}$ VCSEL laser

The phase noise measured with the $1550 \mathrm{~nm}$ single mode VCSEL is depicted in Figure 6. This time, a relatively high phase noise floor can be observed, of about $-120 \mathrm{dBc} / \mathrm{Hz}$, which is much higher than the noise floor which can be computed from the $10 \mathrm{MHz}$ RIN performance of this laser (about $-140 \mathrm{~dB} / \mathrm{Hz}$ ). Up to now, we have no clear explanation of this behavior and further experiments will be performed with this device.

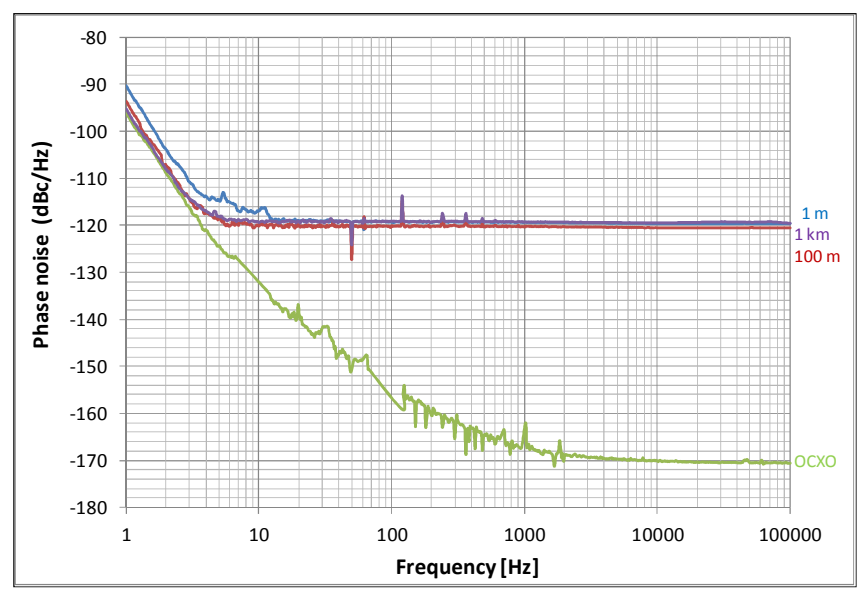

Figure 6. Phase noise at the output of the $10 \mathrm{MHz}$ optical links realized with the $1550 \mathrm{~nm}$ single mode VCSEL

\section{MULTIMODE VCSEL LASER LINK}

The multimode $850 \mathrm{~nm}$ VCSEL (Ulm Photonics) delivers a much higher optical power than the single mode ones. In our experiment, this device was biased at $35 \mathrm{~mA}$, delivering about $4 \mathrm{~mW}$ optical power, and the RF modulation index $m$ was fixed at 0.7 .

The laser has been directly connected to a silicon photodiode (Thorlabs FDS02), using a SMA - FC/PC cable. In another configuration, a $300 \mathrm{~m}$ multimode fiber spool is placed between the laser and the photodiode.

The measured phase noise at the photodiode output is depicted in Figure 7. Although the phase noise shape looks very similar to the one measured with the single mode VCSELs devices, the value of the phase noise floor has been found to be strongly dependent on the bias conditions (it has been optimised in this case down to $-130 \mathrm{dBc} / \mathrm{Hz}$ ). The RIN spectrum shape (not shown here) is also rapidly changing with the bias conditions, probably because of the multimode operation. Therefore, even if the link phase noise performance looks fairly good, we believe that this device is not well fitted for high quality time and frequency applications.

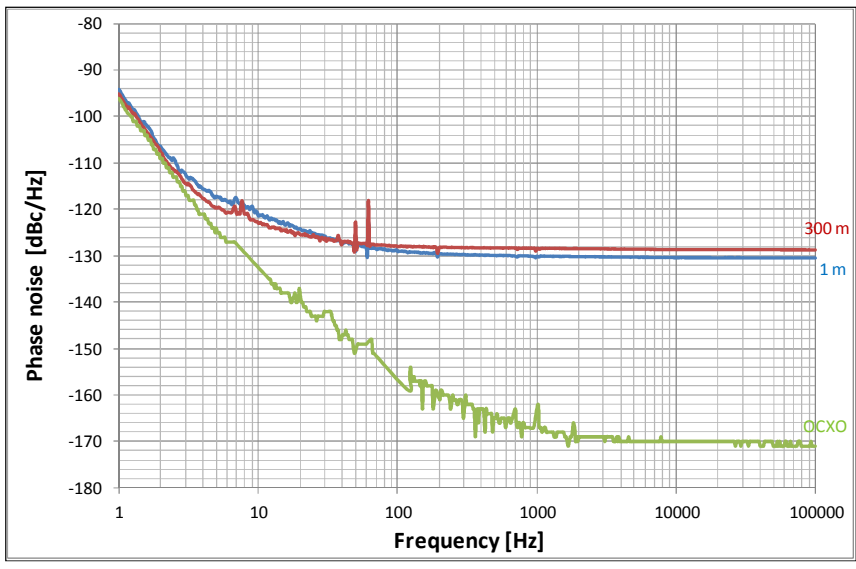

Figure 7. Phase noise at the output of the $10 \mathrm{MHz}$ optical links realized with the $850 \mathrm{~nm}$ multimode VCSEL

Finally, the measured main phase noise data are depicted in Table 2. At $1 \mathrm{~Hz}$ offset, all the lasers are able to transfer the OCXO performance. At $10 \mathrm{~Hz}$ offset, only the DFB laser can keep the OCXO performance, and a degradation of about $10 \mathrm{~dB}$ is observed with all the VCSELs devices. At $100 \mathrm{kHz}$, which corresponds to the noise floor, the best performance is reached with the DFB laser $(-153 \mathrm{dBc} / \mathrm{Hz})$, but this performance is not maintained on long links. A relatively good performance has also been measured with the single mode $1310 \mathrm{~nm}$ VCSEL at this frequency offset (-135 dBc/Hz). 


\begin{tabular}{|c|c|c|c|c|}
\hline $\begin{array}{l}\text { Laser } \\
\text { offset freq } \\
\& \text { link length } \\
\end{array}$ & $\begin{array}{l}\text { DFB } \\
1550 \mathbf{~ n m} \\
\text { Single-mode }\end{array}$ & $\begin{array}{l}\text { VCSEL } \\
\mathbf{1 3 1 0} \mathbf{n m} \\
\text { Single-mode }\end{array}$ & $\begin{array}{l}\text { VCSEL } \\
\mathbf{1 5 5 0} \mathbf{~ n m} \\
\text { Single-mode }\end{array}$ & $\begin{array}{l}\text { VCSEL } \\
\mathbf{8 5 0} \mathbf{n m} \\
\text { Multi-mode }\end{array}$ \\
\hline $\begin{array}{l}\mathbf{L}(f) @ 10 \mathrm{~Hz} \\
\mathrm{~L}=1 \mathrm{~m}\end{array}$ & $-130 \mathrm{dBc} / \mathrm{Hz}$ & $-119 \mathrm{dBc} / \mathrm{Hz}$ & $-119 \mathrm{dBc} / \mathrm{Hz}$ & $-121 \mathrm{dBc} / \mathrm{Hz}$ \\
\hline $\begin{array}{l}\mathrm{L}(\mathrm{f}) @ 100 \mathrm{kHz} \\
\mathrm{L}=1 \mathrm{~m}\end{array}$ & $-153 \mathrm{dBc} / \mathrm{Hz}$ & $-135 \mathrm{dBc} / \mathrm{Hz}$ & $-119 \mathrm{dBc} / \mathrm{Hz}$ & $-130 \mathrm{dBc} / \mathrm{Hz}^{*}$ \\
\hline $\begin{array}{l}\mathrm{L}(\mathrm{f}) @ 10 \mathrm{~Hz} \\
\mathrm{~L}=100 \mathrm{~m}\end{array}$ & $-130 \mathrm{dBc} / \mathrm{Hz}$ & $-119 \mathrm{dBc} / \mathrm{Hz}$ & $-120 \mathrm{dBc} / \mathrm{Hz}$ & $\begin{array}{l}-123 \mathrm{dBc} / \mathrm{Hz} \\
\mathrm{L}=300 \mathrm{~m}\end{array}$ \\
\hline $\begin{array}{l}\mathrm{L}(\mathrm{f}) @ 100 \mathrm{kHz} \\
\mathrm{L}=100 \mathrm{~m}\end{array}$ & $-148 \mathrm{dBc} / \mathrm{Hz}$ & $-136 \mathrm{dBc} / \mathrm{Hz}$ & $-120 \mathrm{dBc} / \mathrm{Hz}$ & $\begin{array}{l}-128 \mathrm{dBc} / \mathrm{Hz}^{*} \\
\mathrm{~L}=300 \mathrm{~m}\end{array}$ \\
\hline $\begin{array}{l}\mathbf{L}(\mathrm{f}) @ 10 \mathrm{~Hz} \\
\mathrm{~L}=1 \mathrm{~km}\end{array}$ & $-130 \mathrm{dBc} / \mathrm{Hz}$ & $-117 \mathrm{dBc} / \mathrm{Hz}$ & $-119 \mathrm{dBc} / \mathrm{Hz}$ & \\
\hline $\begin{array}{l}\mathrm{L}(\mathrm{f}) @ 100 \mathrm{kHz} \\
\mathrm{L}=1 \mathrm{~km}\end{array}$ & $-142 \mathrm{dBc} / \mathrm{Hz}$ & $-131 \mathrm{dBc} / \mathrm{Hz}$ & $-119 \mathrm{dBc} / \mathrm{Hz}$ & \\
\hline
\end{tabular}

Table 2 : Phase noise data measured at $10 \mathrm{~Hz}$ and $100 \mathrm{kHz}$ offset

\section{CONCLUSION}

The investigations described in this paper demonstrate that the medium power DFB laser is still the best device for time\&frequency applications. However, the single mode VCSEL, today available at telecommunications wavelengths, is becoming interesting. It is still limited in signal to noise ratio, but it is reliable enough for high integration time (close to carrier performance) and could be used in clock distribution as an alternative to optical power splitting. Finally, the multimode VCSEL looks interesting at first glance, but the noise parameters are too much sensitive to the bias conditions to recommend this device for high quality analog applications.

\section{ACKNOWLEDGMENT}

This work has been performed in the frame of VERTIGE contract, sponsored by DIRECCTE Midi-Pyrénées, and in relation with Microsystèmes company (Toulouse).

\section{REFERENCES}

[1] R.T Logan, G. F. Lutes, "High stability microwave fiber optic systems", 1992 IEEE Int. Freq. Control. Symp, pp. 310-316.

[2] M.B. Bibey, F. Deborgies, M. Krakowski, D. Mongardien, "Very low phase noise optical links : experiments and theory", IEEE Trans. on Microwave Theory and Tech., vol 47, $\mathrm{N}^{\circ} 12$, dec 1999, pp. 2257-2262.

[3] G. Quadri, H. Martinez-Reyes, B. Benazet, O. Llopis "A Low Phase Noise Optical Link for Reference Oscillator Signal Distribution” Proc. of the IEEE Freq. control Symposium and EFTF, Tampa (Florida), may 2003, pp. 336-340.

[4] G. Santarelli, F. Narbonneau, C. Daussy, O. Lopez, M.E. Tobar, "High performance frequency dissemination for metrology applications with optical fibers", Proc. of the 2005 IEEE Int. Frequency Control Symposium, pp. 925-927.

[5] K.W. Holman, D.J. Jones, D.D. Hudson and J. Ye "Precise frequency transfer through a fiber network using $1.5-\mu \mathrm{m}$ mode-locked sources", Opt. Lett., 29, 1554-1556, 2004.

[6] O. Okusaga, J. Cahill, W. Zhou, A. Docherty, G.M. Carter, C.R. Menyuk, "Optical scattering induced noise in RF-photonic systems," Int. Frequency Control (IFCS) and the European Frequency and Time Forum (EFTF) joint Conference, pp.1-6, May 2011.

[7] G.P. Agrawal, « Fiber-Optic Communication Systems » Wiley Ed., 1997. 